\title{
COMPUESTOS REDUPLICADOS EN EL AIMARA
}

\author{
REDUPLICATED COMPOUNDS IN AYMARA
}

\section{MARCO ANTONIO LOVÓN CUEVA*}

ReSUMEN: Uno de los procesos de formación de palabras es la composición. Este mecanismo morfológico ha sido poco estudiado en las lenguas andinas como el aimara, sobre todo en el caso de los compuestos reduplicados, en el que se unen dos piezas léxicas idénticas para crear un nuevo vocablo. Este artículo tiene por propósito mostrar una descripción de las maneras en que se conforman las palabras compuestas reduplicadas en esta lengua. Metodológicamente, se utilizan los datos procedentes de los principales diccionarios en aimara, así como de los hablantes. Con este trabajo, se concluye que el aimara es una lengua que emplea compuestos reduplicados para formar palabras compuestas básicamente de orden nominal.

Palabras Clave: Morfología, reduplicación, composición, compuesto reduplicado, aimara.

Aвstract: One of the processes of word formation is composition. This morphological mechanism has been little studied in Andean languages such as Aymara, especially in the case of reduplicated compounds, in which two identical lexical pieces are joined to create a new word. This article aims to show a description of the ways in which reduplicated compound words are formed in this language. Methodologically, data from the main Aymara dictionaries and from the speakers are used. With this work, we conclude that Aymara is a language that uses reduplicated compounds to form compound words basically of nominal order.

KEYwORDs: Morphology, reduplication, composition, reduplicated compound, Aymara.

Recibido: 21.01.2020. Aceptado: 27.04.2021.

\footnotetext{
* Magíster en Lingüística. Académico de la Pontificia Universidad Católica del Perú (PUCP), Lima, Perú. Correo electrónico: marco.lovon@pucp.pe. Orcid: https://orcid.org/0000-0002-9182-6072
} 


\section{INTRODUCCIÓN}

C L aimara es la segunda lengua andina más hablada en el Perú. EnCtre los procesos de formación de palabras, sus hablantes emplean los mecanismos de la derivación y la composición, que se presentan en las diversas lenguas del mundo como los procedimientos de formación de palabras más comunes (Almela, 1999; Alvar, 2006; Aronoff, 1976; Bauer, 2009; Booij, 2007; Haspelmath, 2002; Lang, 1992; Lieber \& Štekauer, 2009; Plag, 2003; Sánchez, 2009: Gonzalo, 2018; Spencer \& Zwicky, 2001; Varela, 2005). Una clase de palabras compuestas que aparece en esta lengua son los compuestos reduplicados, en los que se duplica la pieza léxica para crear un nuevo vocablo.

En el caso del quechua, Carreño (2006) ha señalado que esta lengua presenta casos de compuestos reduplicados e indica que "[l]a reduplicación es un proceso muy recurrido en quechua y no se limita a la composición nominal. La raíz reduplicada tiene un sentido colectivo" (p. 10). Algunos ejemplos que cita son los siguientes: wasiwasi 'un conjunto de casas, vecindario', de wasi 'casa' + wasi 'casa' y allpaallpa 'un conjunto de tierras, terral', de allpa 'tierra' + allpa 'tierra'. Por su parte, Weber ([1989] 1996, pp. 417-427) llega a considerar las reduplicaciones como compuestos: "El término 'reduplicación' aquí se refiere a cualquier proceso por el que se repite un elemento -ya sea idéntico o de forma muy semejante, y probablemente acompañado de otros sufijos- para formar un elemento compuesto" (p. 417). El autor ilustra el fenómeno con ejemplos como pakapaka 'especie de ave (considerada de mal agüero)' o mukrumukru 'desigual'.

Respecto del aimara, lengua que investigamos, este mismo procedimiento gramatical ha sido desatendido dentro de las gramáticas escritas de la lengua y sus compendios gramaticales (Hardman, Vásquez y de Dios Yapita, 1988; Deza, 1992; Coler, 2014). En el aimara, las reduplicaciones apenas han sido estudiadas por Briggs (1993), quien señala que "[1]a reduplicación (la repetición) es un proceso bastante productivo en aymara” (p. 262). Al respecto, Briggs indica que existen tres clases de reduplicaciones en la lengua:

1) Raíces nominales reduplicativas, es decir "ciertas raíces nominales [que] no se usan jamás solas, sino reduplicadas" (p. 262).

2) Troncos reduplicativos utilizados como nombres, es decir, "troncos que funcionan como unidades semánticas nuevas con significados derivados de sus raíces bases" (p. 262). 
3) Reduplicación circunstancial, es decir, "las raíces nominales y verbales y ciertos sufijos [que] pueden reduplicarse intermitentemente para dar mayor énfasis. A este proceso lo llamamos reduplicación circunstancial" (pp. 262-263).

Lo que nos dice Briggs es que las reduplicaciones son el resultado de casos de: 1) palabras cuyos constituyentes no aparecen solos (no puede identificarse el significado de sus constituyentes); 2) palabras cuyos constituyentes se reconocen y se usan solos, es decir de forma autónoma o independientemente (puede identificarse el significado de los constituyentes de las palabras reduplicadas); y 3) palabras y sufijos que se reduplican para dar énfasis al discurso, los cuales más bien corresponden a repeticiones. Este último grupo refiere a lo que Roca y Suñer (1998) han denominado reduplicación discursiva. Por tanto, Briggs reúne todos los casos de las reduplicaciones como formas particulares del mismo fenómeno. Ella no establece la diferencia de las expresiones que se forman por cuestiones morfológicas con las que se por forman por cuestiones discursivas.

En este trabajo, por lo tanto, nos interesa estudiar el fenómeno de los compuestos reduplicados en el aimara: su estructura y su semántica. Nuestro propósito es realizar una descripción detenida de las maneras en que se conforman las palabras compuestas reduplicadas en esta lengua. Para ello, se utilizan los datos procedentes de los principales diccionarios de la lengua y de la información ofrecida de los hablantes.

\section{LA COMPOSICIÓN REDUPLICADA Y EL AIMARA}

\subsection{Las reduplicaciones como casos de composición}

En ciertas lenguas existen palabras formadas por la duplicación de un morfema léxico, a las que se les conoce como formas reduplicadas o, simplemente, reduplicaciones. Una muestra de ellas son los casos registrados en (1):

(1) Reduplicaciones en Castellano PERUano y EN QUeChuA

a. Castellano

i. picapica 'confeti o challa'

b. Quechua

i. sach'a sach'a 'arboleda' (Carreño, 2006, p. 45)

ii. unu unu 'inundación’ (Carreño, 2006, p. 45) 
Lo que muestran estos ejemplos son casos de palabras que se han formado por la duplicación idéntica o total de un segmento. Sin embargo, en el proceso de formación de palabras, la reduplicación no siempre termina en una copia idéntica, pues existe otro tipo de reduplicación denominada reduplicación parcial.

Según Inkelas y Zoll (2005, p. 1), la reduplicación es de dos tipos: una total y otra parcial. En la primera ocurre una duplicación completa del lexema copiado, mientras que en la duplicación parcial se copia solamente una parte de un lexema. Por ello, el resultado de la reduplicación total se le conoce como copia perfecta, mientras que el de la reduplicación parcial como copia imperfecta o copia similar. Estos autores proponen los siguientes ejemplos, tomados de tres lenguas diferentes, para ilustrar esta clasificación.

(2) REDUPLiCACIONES PARCialeS y totales

\begin{tabular}{|c|c|c|c|c|}
\hline kira: & 'llamada' & $\rightarrow$ & kik-kira: & (llamadas continuas) \\
\hline $\begin{array}{l}\text { b. Amele } \\
\text { balado-? }\end{array}$ & 'estropear' & $\rightarrow$ & bala-bulu-do? & (llorar mucho) \\
\hline $\begin{array}{l}\text { c. Warlpiri } \\
\text { Kamina }\end{array}$ & 'señorita' & $\rightarrow$ & kamina-kamina & (plural) \\
\hline
\end{tabular}

(Inkelas y Zoll, 2005, p. 1)

Observando estos ejemplos, la copia fonética presente en (2c) es perfecta, mientras que las de (2a) y (2b) no. En (2a), hay una vocal que no se copia, tampoco una consonante copia la consonante de ataque (r) de la segunda sílaba, y, en (2b), las vocales de la copia no son las mismas de la base léxica de la que deriva la duplicación; mientras que en (2c) se copian perfectamente todos los fonemas. En otras palabras, (2a) y (2b) son casos de reduplicaciones parciales; por el contrario, (2c) es un caso de reduplicación total.

Como las reduplicaciones tienen en común con las palabras compuestas el componerse por la adjunción de lexemas, para algunos lingüistas, las reduplicaciones son casos de composición morfológica (Roca y Suñer, 1998, p. 44), es decir clases de compuestos, porque por sus características parecen presentar los mismos comportamientos del proceso de composición (Štekauer, Valera y Körtvélyessy, 2012). Así, Fabb (2001) indica que una de 
las clases de compuestos son los compuestos por repetición. El autor señala que la reduplicación de palabras se describe como un proceso de composición, porque cada parte de la palabra resultante corresponde a una palabra independiente (Fabb, 2001, p. 69).

Sin embargo, dicho estatus es discutido por la teoría lingüística, pues se debate si la reduplicación es un fenómeno morfológico distinto o nuevo, o si es un tipo de derivación, o si es una clase de composición tal como creemos y sostenemos. Para algunos teóricos, se trata de un fenómeno diferente o particular de formación de palabras; dicho de otro modo, la reduplicación es un fenómeno distinto a otros procesos de formación de palabras. Una manera que se emplea para distinguir entre composición (o cualquier otro proceso) y reduplicación es la que refiere al resultado fonológico o asociación fonológica: se sostiene que, en la primera, la forma es distinta, mientras que, en la segunda, las piezas léxicas son similares; por eso, la reduplicación es tratada como una estrategia lingüística que difiere de la composición o cualquier otro mecanismo. También, ha sido equiparable con el fenómeno de la derivación morfológica, en tanto que parece que un afijo reduplicado deriva nuevas categorías distintas a las de sus constituyentes (Inkelas, 2014, p. 174). En relación con ello, se sostiene que se reduplica un afijo y se da paso a una derivación (Plag \& Winther, 2016). En cambio, otros teóricos consideran que se reduplica un lexema, y no un afijo, pues no presenta el comportamiento de un afijo, y se da paso a la composición. En otro sentido, la reduplicación es un tipo de composición, en la que opera el proceso de adjunción de lexemas para formar una nueva palabra. En general, la reduplicación se considera un tipo de proceso morfológico noconcatenativo (o metamórfico), que modifica la base misma, como ocurre con las reduplicaciones parciales, más que agregar un segmento. No obstante, la tesis contraria sostiene que se trata de la adjunción de palabras, en casos en que ocurre una reduplicación total (y exacta). Para nosotros, las reduplicaciones morfológicas en el aimara, es decir, en las que se combinan dos palabras para formar una nueva, son casos de composiciones morfológicas, en tanto que parecen seguir la misma operación morfológica que las composiciones.

Asumiendo entonces que las reduplicaciones y las composiciones presentan características comunes, una de las propiedades en común sería que las formaciones con reduplicación siempre dan lugar a una pieza léxica nueva con especificaciones semánticas y categoriales propias, como sucede con el caso de los compuestos. Así, por ejemplo, en el caso de la palabra picapica, esta parte de una forma verbal (el verbo picar) para generar un sustantivo con un valor semántico particular: 'confeti', en el castellano pe- 
ruano. Es decir, se genera una nueva palabra con sus propias categorías y significados. Es por ello por lo que a las reduplicaciones morfológicas se les ha llegado a conocer como compuestos reduplicados (Roca y Suñer, 1998), reduplicaciones por composición (Fabb, 2001) o compuestos reduplicativos.

En conclusión, los compuestos reduplicados son piezas léxicas con una especificación semántica y sintáctica única formados a partir de la copia fonológica de una palabra existente en la lengua. En otro sentido, un ítem lexical puede reduplicarse para formar un compuesto con un significado nuevo, que se distingue de sus partes, las cuales se combinan para formar una nueva unidad léxica con una categoría gramatical propia.

Ahora bien, según Roca y Suñer (1998, p. 44), este tipo de reduplicación a la que denomina reduplicación morfológica se diferencia tanto de la reduplicación léxica, que opera en una oración para dar valor de intensificación o cuantificación sobre una propiedad, acción o evento sin formar una nueva palabra o vocablo -que se distingue de aquellas reduplicaciones que sí forman palabras nuevas con valores de intensidad, cantidad, etc.-, así como de la reduplicación discursiva, que es utilizada para repetir una unidad que debe dar valor enfático a todo el enunciado, y en la que tampoco se forma una nueva palabra, tipologías que se ilustran en (3) y (4) respectivamente.

\section{(3) EJEMPLOS DE REduplicaCióN LÉXICA}
a. Quiero café café. (Roca y Suñer, 1998, p. 45)
b. El postre estaba rico rico. (Roca y Suñer, 1998, p. 45)

\section{(4) EJEMPLOS DE REDUPLICACIÓN DISCURSIVA}

a. ¿YY todavía quiere que le pague? Jamás, jamás, jamás. (Roca y Suñer, 1998, p. 45)

b. ¡Cállate, hombre, cállate! (Roca y Suñer, 1998, p. 45)

Como se ve en (3), en la reduplicación léxica se repite literalmente uno de los constituyentes sintácticos con fines expresivos de intensificación en una acumulación de significantes sin que medie alguna pausa entre ellos y sin dar paso a una unidad morfológica o a la formación de una nueva palabra. Paralelamente, también, como se ve en (4), en la reduplicación discursiva se reitera más de una vez un lexema por motivos pragmáticos, generalmente para expresar énfasis o insistencia, en la que dichos lexemas aparecen separados por una pausa o una ruptura en la entonación, o inclu- 
so por alguna palabra. Sin embargo, como puede deducirse de la explicación, ni la reduplicación léxica ni la así llamada reduplicación discursiva crean palabras nuevas como sí lo hacen las reduplicaciones morfológicas o compuestos reduplicados.

\subsection{Los compuestos reduplicados en lengua aimara}

El aimara presenta compuestos reduplicados bajo la forma de reduplicaciones totales. Las siguientes reduplicaciones muestran la duplicación total de los lexemas copiados perfectamente para crear nuevas palabras, como se muestra en (5).

(5) Reduplicación total en Aimara

a. qala qala piedra piedra 'pedregal'

b. awki awki anciano anciano 'danza satírica sobre ancianos españoles'

Tanto (5a) como (5b) muestran la duplicación de lexemas nominales, como se muestra en (6). Para (5a), la secuencia silábica CV.CV se copia; mientras que para (5b), se copia la secuencia VC.CV, como se muestra a continuación.

(6) REPRESENTACIÓN FONOLÓGICA DE LA REDUPLICACIÓN

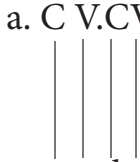

q a. la

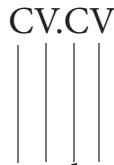

qa. la

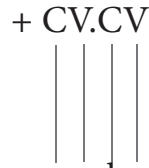

qa. la

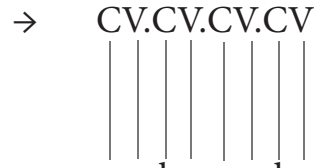

qa. la. qa. la

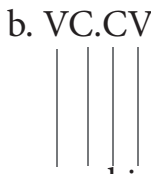

aw. ki
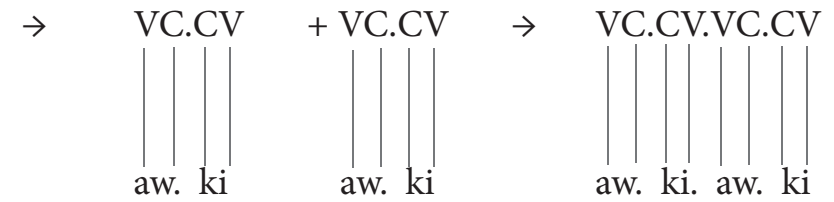
Esta representación autosegmental nos muestra las secuencias silábicas y la presencia de resilabificaciones que se generan en la formación de palabras compuestas. En esta ilustración, se ve cómo una palabra (qala 'piedra' o awki 'anciano') se reduplica totalmente para favorecer la creación de un compuesto reduplicado (qalaqala 'pedregal' y awkiawki 'danza en la que se imita a los ancianos' $)^{1}$.

Cabe señalar que una diferencia semántica entre el compuesto reduplicado (6a) y el (6b) es que el primero es un caso de composicionalidad y el segundo no. El significado del compuesto reduplicado (6a) es producto de la suma de las partes: qala 'piedra' + qala 'piedra' da por resultado qalaqala 'pedregal'; en este caso, los constituyentes aportan directamente al significado total del compuesto reduplicado, mientras que el significado de (6b) está subcategorizado o especificado, en el sentido de que el significado del compuesto no deriva necesariamente de la suma de las partes, sino de una interpretación semántica, como si se trata de un compuesto exocéntrico: awki awki no es la suma de 'viejo' más 'viejo'; es decir, el significado literal "los viejos" no es el origen de la danza. Su origen no es composicional y tal significado no se ha aplicado para designarla.

\subsection{La lengua aimara}

El aimara es una de las lenguas andinas más importantes (Andrade, 2009), por el número de sus hablantes, el uso y prestigio de la lengua y el número de investigadores que la estudian. Esta se habla en casi toda la meseta del Collao y en las zonas que sus hablantes han migrado. Los países en que aún se habla la lengua son Perú, Bolivia, algunas zonas al norte de Chile (Arica, Iquique, Antofagasta), y en las provincias de Salta y Jujuy en Argentina. Es la segunda más hablada en los Andes, después del quechua (unos 1.920.458 hablantes, según Gonzalo, 2011).

En la actualidad, los hablantes aimaras son, en su mayoría, bilingües aimara-castellano. Por la influencia y prestigio del castellano, en algunas zonas se va perdiendo la lengua (Cerrón-Palomino y Carvajal, 2013, pp. 170-171), por lo que hay zonas donde se aprende como segunda lengua (Lovón, 2016). Además, muchos hablantes del aimara, como los del que-

\footnotetext{
${ }^{1}$ Según Cotari, Mejía y Carrasco (1978, p. 43), awki awki se define como "danza folklórica 'los viejitos'. Los danzantes se visten de viejos con joroba, una chaqueta con leva larga, sombrero de copa, unos anteojos, una careta con barba larga y un bastón torcido. De esta manera ridiculizan a los eminentes españoles de la época del coloniaje. / 'Aka phistanjj awki awkiw utjan" siwa. Dice que en esta fiesta habrá la danza 'awki awki”'.
} 
chua (Lovón, 2020) o el jaqaru (Lovón, 2009), es decir de las otras lenguas andinas en el Perú, son marginados social y económicamente por los peruanos (Lovón y Quispe, 2020), por lo que repercute en su decisión de dejar de hablar y trasmitir la lengua. Las lenguas andinas no son valoradas en el mundo occidental (Lovón, 2018). Sin embargo, sus hablantes luchan por mantener y usarlas (De Pedro Ricoy, Howard, Reynoso y Andrade, 2020).

Los primeros estudios sobre la lengua han sido realizados por Bertonio ([1612] 1984); posteriores estudios han analizado su fonología, morfología y sintaxis (Cerrón-Palomino, 2000). La lengua posee 3 vocales (la vocal alta anterior /I/, la vocal alta posterior $/ \mathrm{v} /$ y la vocal baja central $/ \mathrm{a} /$ ) y 26 consonantes $\left(/ \mathrm{p} /, / \mathrm{p}^{?} /, / \mathrm{p}^{\mathrm{h}} /, / \mathrm{t} /, / \mathrm{t}^{?} /, / \mathrm{t}^{\mathrm{h}} /, / \mathrm{k} /, / \mathrm{k}^{?} /, / \mathrm{k}^{\mathrm{h}} /, / \mathrm{q} /, / \mathrm{q}^{?} /, / \mathrm{q}^{\mathrm{h}} /, / \mathrm{c}^{2} /, / \mathrm{c}^{?} /, / \mathrm{c}^{\mathrm{h}} /\right.$, $/ \mathrm{s} /, / \chi /, / \mathrm{h} /, / \mathrm{m} /, / \mathrm{n} /, / \mathrm{h} /, / \mathrm{l} /, / \lambda /, / \mathrm{r} /, / \mathrm{w} /, / \mathrm{y} /)$. Es una lengua polisintética, aglutinante y sufijante (no existen prefijos, los afijos se ordenan postpuestos a una raíz). Presenta palabras derivadas y compuestas (Gonzalo, 2011, Lovón, 2019) y es de tipo SOV (Huayhua, 2001).

\section{METODOLOGÍA}

Este es un estudio descriptivo. Se han recogido los datos de los diccionarios de las variedades lingüísticas bolivianas y peruanas (Ayala, 1988, Büttner y Condori, 1984, Callo, 2009, Carvajal, Hernández y Ramos, 2001, Cotari, Mejía y Carrasco, 1978, De Lucca, 1987, Deza, 1989, Huayhua, 2009) y, particularmente, de la variedad aimara de Conima, del trabajo de campo realizado en la comunidad en el año 2014.

La variedad de Conima es uno de los dialectos aimaras que se localiza en la provincia de Moho, departamento de Puno, en el sudeste del Perú y cuenta aún con hablantes monolingües (es apreciada como una de las variedades menos afectadas por el contacto con el castellano). Respecto de sus hablantes, se estima que hay entre 3000 a 4000 hablantes en Conima. Los pobladores se dedican a la ganadería y la agricultura.

\section{ANÁLISIS}

\subsection{Caracterización de los compuestos reduplicados en el aimara}

Según Adelaar \& Muysken (2004, p. 296), la existencia de elementos reduplicados que juntos constituyen una única raíz ya fue observada por Ber- 
tonio. En una de sus glosas, señala que la palabra huarahuara [warawara] 'estrella' no es un sustantivo que muestre solo repetición, sino que es un sustantivo único. Bertonio ya empezaba a distinguir reduplicaciones que se constituían como una sola raíz o unidad, con un significado no siempre predecible. En otro sentido, las reduplicaciones morfológicas ya se percibían como palabras autónomas, las cuales para nosotros corresponden a casos de palabras compuestas. Por eso, a continuación, teniendo en cuenta los criterios de reconocimiento de los compuestos morfológicos, argumentaremos que las reduplicaciones se comportan como casos de composición.

\subsubsection{Poseen un solo significado}

Las palabras compuestas, sean transparentes u opacas, adquieren un significado nuevo y propio, distinguiéndose del de sus constituyentes (Dressler, 2007). Lo mismo ocurre con las reduplicaciones: estas adquieren nuevos y propios significados, tal como se ilustra a continuación.

(7) Ejemplos DE REDUPLiCACIONES
a. chhiya 'unidad de medida formada por el dedo pulgar y el meñique extendidos'
b. chhiya chhiya 'gusano de tierra, de color verde claro, que se arrastra
como si estuviera midiendo el espacio'
c. jinchu 'oreja, oído'
d. jinchu jinchu 'planta medicinal para bajar las hinchazones'
e. jinq'i 'onomatopeya del sonido que produce el pájaro jinq'i jinq'i
f. jinq'i jinq'i 'lechuza'
g. jinq'a '[or. inc., probablemente protesta]'
h. jinq'a jinq'a 'viento con remolino'
i. waru 'camellón, disposición del suelo en forma de lomadas donde se coloca los cultivos, rodeado con agua'
j. waru waru 'conjunto de camellones'

Como se ve, las palabras simples presentan un significado diferente de las palabras reduplicadas. Es decir, los compuestos reduplicados adquieren un significado nuevo. Dicho de otro modo, teniendo en cuenta este criterio, las reduplicaciones se comportan como palabras compuestas. 


\subsubsection{Poseen un solo acento}

Las palabras compuestas presentan un solo acento; lo mismo ocurre con las reduplicaciones, las cuales poseen un solo acento, que se ubica en la posición penúltima de la palabra, igual que cualquier palabra simple o compuesta de la lengua, como se muestra en (8). Observemos los siguientes casos:

(8) ACENTo En las PAlabras Simples y EN LAS REDUPliCACiONES
a. ['čhiya] <chhiya $>$
f. [č́n iya' č̀hiya]
$<$ chhiyachhiya $>$
b. ['henčo] <jinchu>
g. [henčð'hejčð]
$<$ jinchujinchu $>$
c. ['henq? $\varepsilon] \quad<$ jinq'i $>$
h. [henq? $\varepsilon^{\prime}$ henq? $\left.\varepsilon\right]$
$<$ jinq'ijinq'i $>$
d. ['henq?a] <jinq'a $>$
i. [henq?a'henq?
e. ['waru]
j. [waru'waru]
$<$ jinq'ajinq'a $>$
$<$ waruwaru $>$

Los ejemplos muestran que las reduplicaciones morfológicas portan un único acento, como las palabras simples y compuestas. Podemos concluir entonces que las reduplicaciones morfológicas se comportan como cualquier palabra simple: no solo por presentar un significado único, sino también por contar con un acento tónico único asignado. Lo mismo ocurre con las palabras compuestas, que también hacen referencia a una entidad distinta a la de las partes y poseen un solo acento tónico. En (9) se comparan reduplicaciones con composiciones:

(9) Reduplicaciones y COMPUESTOS: Un SOLO ACENTO

Formas reduplicadas
a. [č $\check{c}^{h}$ iya' ' ${ }^{h}$ iya $]$
'gusano de tierra, de color verde claro'
c. [henq? $\left.\varepsilon^{\prime} h e n q ? \varepsilon\right]$
'lechuza'
e. [hejčð'hejčð]
'planta medicinal'

Formas compuestas
b. [amay'laqO] 'gusano de color negro'
d. [amayka' $\lambda \mathrm{apu}]$ 'ataúd'
f. [hejčð'lik?i] 'cerumen'

El paralelismo nos demuestra que las reduplicaciones morfológicas comparten las mismas propiedades fonológicas (un acento tónico) e incluso semánticas (un significado propio) que definen a las palabras compuestas, lo que nos da pie para considerarlas, una vez más, como casos de composición. Es decir, estas palabras cumplen con los mismos criterios de 
reconocimiento de un compuesto: poseen un solo acento y aluden a un referente conceptual único.

\subsubsection{Poseen cohesión sintáctica}

Las palabras compuestas son piezas sintácticas sólidas, que pertenecen a una categoría gramatical específica, y que pueden ser objeto de flexión y derivación, o de cualquier otra modificación morfosintáctica, sin que estas alteren su cohesión formal.

En relación con ello, en primer lugar, podemos señalar que las reduplicaciones morfológicas, al igual que las palabras compuestas, también son unidades sintácticas, las cuales, por ejemplo, impiden que se intercale algún morfema en su interior, descomponiendo su unidad morfosintáctica. Como podemos ver en (10), la introducción de morfemas dentro de las formas compuestas ocasiona casos de agramaticalidad o ambigüedad, pues su descomposición daría paso a interpretaciones léxicas y hasta discursivas relativamente aceptables en detrimento de la lectura de los compuestos reduplicados. Recordemos que en las reduplicaciones léxicas se duplican dos palabras que mantienen su autonomía (Escandell, 1991), por lo que una de ellas se puede eliminar sin mayores consecuencias, mientras que, en el caso de los compuestos reduplicados, no puede ocurrir porque los lexemas se encuentran fundidos en una sola palabra, y si ocurriese, el significado al que hacen referencia se alteraría o se obtendrían formas ininteligibles. A continuación, observemos cómo pueden verse afectados los compuestos jinchujinchu 'planta medicinal para bajar las hinchazones' y chhiyachhiya 'gusano de tierra' con la inserción de morfemas.

(10) IMPOSIBILIDAD DE INSERCIÓN DE MORFEMAS AL INTERIOR DE UN COMPUESTO REDUPLICADO
a. $\quad{ }^{*}[\mathrm{jinchu}]_{\mathrm{N}} \quad[-1 \mathrm{la}] \quad[\mathrm{jinchu}]_{\mathrm{N}} \quad$ 'lit. oreja orejita'
b. ${ }^{*}[\mathrm{jinchu}]_{\mathrm{N}} \quad[-\mathrm{ni}] \quad[\mathrm{jinchu}]_{\mathrm{N}} \quad$ 'lit. oreja, su orejita'
c. ${ }^{*}[\text { jinchu }]_{\mathrm{N}} \quad[$-rara $] \quad[\mathrm{jinchu}]_{\mathrm{N}} \quad$ 'lit. oreja, orejas'
d. ${ }^{*}\left[\right.$ chhiya $_{\mathrm{N}} \quad[\text { jach'a }]_{\mathrm{Adj}} \quad[\text { chhiya }]_{\mathrm{N}} \quad$ 'lit. lejos, bastante lejos'
e. ${ }^{*}\left[\right.$ jinq'a $_{\mathrm{N}} \quad[\text { jach'a }]_{\text {Adi }} \quad[\text { jinq'a }]_{\mathrm{N}} \quad$ 'lit. protesta, bastante protesta'

Como se muestra en los casos (10a), (10b) y (10c), un morfema derivativo como -lla 'diminutivo', -ni 'posesivo', o -rara 'cuantitativo', o una raíz 
léxica como jacha 'bastante' en (10d) y (10e) son imposibles de insertarse dentro del compuesto reduplicado sin que se vea afectado, probando que la lengua no admite inserciones dentro de un compuesto reduplicado, es decir, al interior de una palabra compuesta. En el primer caso, los sufijos derivativos solo pueden adherirse a núcleos -o unidades léxicas (por ejemplo, las siguientes formas son gramaticales y aceptables en la lengua: utalla 'casita', wankani 'lugar que tiene roca sagrada', anurara 'con muchos perros')-, y como la forma reduplicada funciona como una sola palabra, ellos deben aparecer al final de esta, no en su interior; en el segundo caso, el modificador jachá, que se ha insertado también internamente, está alterando la unidad sintáctica, pues los modificadores deben afectar al todo y no a una de las partes. Para que resulten gramaticales, los sufijos deben adherirse al final de la palabra, como se ve en (11a), (11b), (11c), y el modificador debe ocurrir antes de la pieza modificada, como se ve en (11d) y $(11 \mathrm{e})^{2}$.

\section{(11) Relaciones gramaticales}
a. jinchujinchulla
'plantita medicinal'
b. jinchujinchuni
'lugar que posee esas plantas medicinales'
c. jinchujinchurara 'con muchas de esas plantas medicinales'
d. jach'a chhiyachiya 'gusano de tierra grande'
e. jach'a jinq'ajinq'a 'tremendo viento con remolino'

Obsérvense los siguientes ejemplos de compuestos reduplicados en una oración:

\section{(12) Oraciones contextuales}

a. Uka jach'a chhiyachhiyaxa k'achatha sari. \{rka hač?a čn Iyačh Iyax(a) k?ača-t ${ }^{\mathrm{h}}(\mathrm{a}) \quad$ sar(a)-I\} esa grande gusano-de-tierra-TOP despacio-ABL ir-3S

'Ese gran gusano de tierra va despacio.

$\begin{array}{llll}\text { b. Arumaxa } & \text { ch'iyara } & \text { jinq'ijinq'iwa } & \text { uñasini. } \\ \text { \{aroma- } \chi(a) & \text { č? Iyar(a) } & \text { hInq?'IhInq'I-w(a) } & \text { ona-sI-n(I)-I } \\ \text { noche-TOP } & \text { negro } & \text { lechuza-FOC } & \text { aparecer-CONT-LOC-3S }\end{array}$ 'Esa noche aparece la lechuza negra'.

\footnotetext{
${ }^{2}$ En estos casos marcamos las palabras compuestas reduplicadas en cursiva para que se evidencie su unidad sintáctica.
} 
c. Jinchujinchuxa

$\{$ hInčðhInč

planta medicinal-TOC pampana

pampa-n(a)

llanura-LOC ali.

al-I\}

crecer-3S

'La planta medicinal crece en la pampa'.

De esta manera, vemos que los constituyentes están soldados porque conforman una sola unidad léxica; el mismo compuesto impide la separación morfológica. Por ejemplo, la modificación adjetiva nos ayuda a comprender que nos enfrentamos a una palabra y no a dos, ya que esta impide que se modifique un solo constituyente (el adjetivo califica a una pieza sustantiva), como se ilustra a continuación en (13), donde el numeral kimsa 'tres' debe modificar todo el compuesto reduplicado, a todo el nombre (N), y no solo a una de las partes.

\section{(13) IMPosibilidAd DE MOdificAR A UN SOLO CONSTITUYENTE}

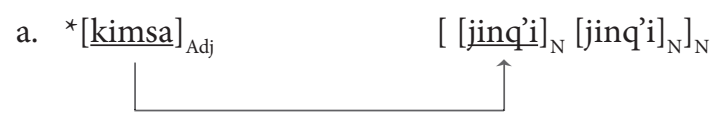

'lit. tres pus, pus

b. $[\underline{\text { kimsa }}]_{\text {Adj }}$ $\left[\text { [jinq'i }_{\mathrm{N}}\left[{ }_{\mathrm{Ninq}} \mathrm{i}\right]_{\mathrm{N}}\right]_{\mathrm{N}}$

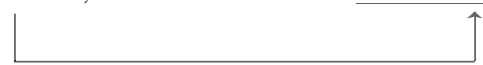

'Tres lechuzas'

c. ${ }^{*}[\text { kimsa }]_{\mathrm{A} d}$

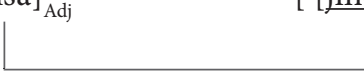

'lit. tres orejas, oreja'

d. $[\underline{\text { kimsa }}]_{\mathrm{Adj}}$

$\left.[\text { [jinchu }]_{\mathrm{N}}[\text { jinchu }]_{\mathrm{N}}\right]_{\mathrm{N}}$

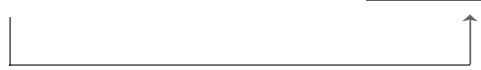

'Tres plantas medicinales'

Como se ilustra, es agramatical que kimsa modifique una sola parte del compuesto reduplicado jinq'i jinq'i y también una parte de jinchu jinchu, porque la modificación adjetiva afecta a todo el compuesto reduplicado. 
En estos ejemplos, podemos ver que el adjetivo ubicado antes del sustantivo, es decir el modificador antes del núcleo, sigue el patrón sintáctico de la lengua; en otro sentido, la dirección de la modificación respeta el orden MODIFICADOR MODIFICADO y no otro. De todo esto, podemos concluir que los compuestos reduplicados son estructuras lingüísticas independientes que poseen una categoría gramatical determinada (en estos casos $\mathrm{N}$ ) y que se ven afectados por otras piezas léxicas con sus propias categorías (como es el adjetivo).

\subsection{Semántica de los compuestos reduplicados}

Respecto del valor semántico de las reduplicaciones morfológicas, Roca y Suñer $(1998$, p. 43) señalan que estas pueden referirse a pluralidad, usos distributivos o cuantificación, intensificación, o variaciones aspectuales (por lo general, aspecto durativo o iterativo), como se ve en (14):

(14) VALOR SEMÁNTICo DE LAS REDUPLICACIONES

\begin{tabular}{|c|c|c|c|c|c|}
\hline \multicolumn{6}{|l|}{ a. plural } \\
\hline anak & 'niño' & $\rightarrow$ & anakanak & 'niños' & (malayo) \\
\hline \multicolumn{6}{|c|}{ b. cuantificación } \\
\hline bar & 'dos' & $\rightarrow$ & barbar & 'los dos' & (tzeltal) \\
\hline \multicolumn{6}{|c|}{ c. aumentativo } \\
\hline dolu & 'lleno’ & $\rightarrow$ & doludolu & 'muy lleno' & (turco) \\
\hline \multicolumn{6}{|c|}{ d. intensificación (diminutivos) } \\
\hline xóyamac & 'niño’ & $\rightarrow$ & xoyamacxóyamac & 'niño pequeño' & (nez percé) \\
\hline \multicolumn{6}{|l|}{ e. aspecto } \\
\hline Eve & 'hablar' & $\rightarrow$ & eveeve & 'hablar mucho' & (samoano) \\
\hline
\end{tabular}

(Roca y Suñer, 1998, p. 43)

Lo propio ocurre en el aimara, pues tenemos compuestos reduplicados que especifican o indican forma, intensificación, entre otros aspectos, como se describe a continuación.

\subsubsection{Compuestos reduplicados de cantidad}

Los compuestos reduplicados de cantidad describen propiedades acumulativas del referente expresado por el elemento duplicado. Según Adelaar \& 
Muysken (2004, pp. 295-296), la reduplicación de sustantivos para indicar una cantidad dispersa es la más frecuente. Los siguientes datos ejemplifican casos de compuestos reduplicados de cantidad.

\section{(15) Compuestos Reduplicados De CANTIDAd}
a. uta uta
casa casa
'caserío'
b. qala qala
piedra piedra
'pedregal'
c. sulla sulla
rocío rocío
'lugar donde abunda rocío'
d. quqa quqa
árbol árbol
'bosque'
e. qullu qullu
cerro cerro
'lugar donde hay muchos cerros'
f. pata pata
andén andén
'andenería'
g. puti puti
cueva cueva
'cueverío'

Estos compuestos evocan ideas de cantidad, conjunto, agrupación, demasía, al señalar por ejemplo 'conjunto de casas', o 'lugar con bastante rocío'. Las siguientes oraciones ilustran los casos de reduplicaciones por cantidad.

(16) LOS COMPUESTOS REDUPLICADOS DE CANTIDAD EN UNA ORACIÓN
a. Lunthata utauta
t'ijukipi.
\{lont thata $^{\text {hat }}$
vtaut(a)-ø
t?'Ihช-kIp(a)-I\}
ladrón
caserío-AC
correr-ATR-3S
'El ladrón corrió atravesando el caserío'. 
b. Allqamari qalaqalaruwa puri.

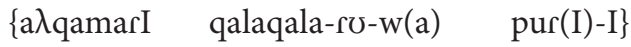

águila pedregal-IL-FOC llegar-3S

'El águila posa sobre el pedregal'.

c. Anu sullasullana sunt'isi.

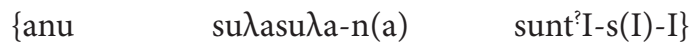

perro rocío-LOC revolcarse-BEN-3S

'El perro se revuelca entre las plantas con mucho rocío'.

d. Jumaxa quqaquqana arch'ukisi.

\{homa- $\chi$ a quqaquqa-na $\quad \operatorname{ar}(\mho)$-č? $v k I-s(I)-I\}$

2S-TOP bosque-LOC cantar-FREC-CONT-3S

'Tú cantas en el bosque'.

e. Qamaqi qulluqulluna utjasi.

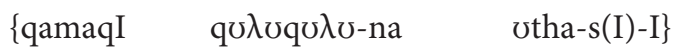

zorro montaña-LOC vivir-BEN-3S

'El zorro vive entre la montaña'.

f. Anuxa patapatana uma laqhuski.

$\left\{\operatorname{an} \mho-\chi(\alpha) \quad\right.$ patapata-n(a) $\quad$ om(a)- $\left.\varnothing \quad \operatorname{laq}^{\mathrm{h}}-\mathrm{sk}(\mathrm{a})-\mathrm{I}\right\}$

perro-TOP andenería-LOC agua tomar-PROG-3S

'El perro está tomando agua en los andenes'.

g. Qamaqi putiputiruwa chhaqi.

\{qamaqI potIpotI-ro-w(a) ch $\left.{ }^{\mathrm{h}} \mathrm{aq}(\mathrm{a})-\mathrm{I}\right\}$

zorro cueverío-IL-FOC perderse-3S

'El zorro se perdió entre las cuevas'.

4.2.2. Compuestos reduplicados de intensidad

Las reduplicaciones de intensidad se producen para señalar propiedades de intensificación o exageración. A continuación, se presentan tres ejemplos que dan cuenta de este tipo de conceptualización.

(17) Compuestos Reduplicados De INTENSIDAd

a. k'aja k'aja

'tos' 'tos'

'tos convulsiva' 

b. lliphi lliphi
'brillo' 'brillo'
'relámpago'

Estos compuestos expresan intensidad semántica de grado mayor al que expresan sus constituyentes. Así, k'ajak'aja 'tos convulsiva' expresa mayor intensidad que k'aja 'tos', como se muestra en (17a); y lliphilliphi 'relámpago' expresa mayor intensidad que lliphi 'brillo', como se registra en (17b). En las siguientes oraciones, se muestra el uso de estos compuestos reduplicados de intensidad.

(18) LOS COMPUESTOS REDUPLICADOS DE INTENSIDAD EN UNA ORACIÓN

a. Aynachana k'ajaḱaja usuwa sarti.

\begin{tabular}{|c|c|}
\hline ača-n(a) & k'ahak'ah(a) \\
\hline & tos.convulsiva \\
\hline
\end{tabular}

'En la selva surge la tos convulsiva'.

$\begin{array}{llll}\text { b. Jallu } & \text { pachana } & \text { lliphilliphiwa } & \text { utji. } \\ \text { hha } \lambda \mho & \text { pača-n(a) } & \lambda \mathrm{Ip}^{\mathrm{h}} \mathrm{I} \lambda \mathrm{Ip}^{\mathrm{h}} \mathrm{I}-\mathrm{wa} & \text { oth }(\mathrm{a})-\mathrm{I}\} \\ \text { lluvia } & \text { tiempo-LOC } & \text { relámpago-FOC } & \text { haber-3S }\end{array}$

'En la época de lluvias hay relámpago'.

\title{
4.2.3. Compuestos reduplicados de semejanza o analogía
}

Los compuestos reduplicados de semejanza o analogía se producen para describir similitudes, apariencias o parecidos conceptuales. Los siguientes ejemplos son casos de este tipo de categorización.

\section{(19) Compuestos Reduplicados De SEMejanza o ANAlogía}

\author{
a. kacha kacha \\ paso paso \\ 'piernas entreabiertas'3
}

\footnotetext{
${ }^{3}$ Cotari, Mejía y Carrasco (1978, p. 148) definen kacha kacha como "relativo a las piernas entreabiertas. Se dice de las personas que caminan lentamente y con las piernas entreabiertas / ¡Kacha kachapí sarnaqasktajja! Janit sum sarnaqañ puirkta? ¡Estás caminando con las piernas entreabiertas! ¿No puedes caminar bien?”.
} 
b. qawra qawra

llama llama

'planta de hoja parecida a las huellas de la pisada de la llama'

Estos compuestos aluden a forma, materia, materialización, composición, estructura. En estos casos, se puede recurrir a la forma que toma un objeto o al parecido conceptual que tiene un objeto con otro.

Por ejemplo, la alusión a la forma de caminar se puede extender a la forma en que uno camina, como se muestra en (19a); o la evocación a un animal, en este caso el auquénido llama, puede aplicarse para referir a una planta cuya hoja guarda un parecido con la forma de la pisada del animal, como se muestra en (19b).

Las siguientes oraciones muestran el uso de estos compuestos reduplicados de semejanza o analogía. Su aparición en una oración nos evidencia, por un lado, que estamos frente a formas compuestas, pues cumplen con las características de un compuesto, y, por otro lado, como sucede con todo compuesto, estos poseen un significado: estas unidades expresan semánticamente alguna propiedad vinculada con el concepto de forma.

(20) LOS COMPUESTOS REDUPLICADOS DE SEMEJANZA EN UNA ORACIÓN
a. Uka jaqixa
kachakacha
sartixa.
\{uka haqI- $\chi(\mathrm{a})$
kačakača
$\operatorname{sar}(a)-t(a)-I-\chi a\}$
ese hombre-TO
piernas.entre.abiertas
ir-ASC-3S-TOP
'Ese hombre camina con las piernas abiertas'.
b. Naya qawraqawra qulluna
\{naya qawraqawr(a)-ø qu $\lambda \mho-n(a)$
is planta-AC cerro-LOC
uñjaniwaytha.
onha-nI-way(a)-th ${ }^{\mathrm{h}}$ \}
ver-CL-INCD-1S
'Yo vi en el cerro la planta llamada qawraqawra'.

Asimismo, las onomatopeyas -o reduplicaciones onomatopéyicas- también expresan conceptualizaciones de semejanza porque reiteran la manera en que se producen los sonidos. Se tratan de casos de repeticiones idénticas de un elemento formal que a pesar de que sus partes no existen como lexemas propiamente en la lengua llegan a tener estatus léxico, es decir, poseen significado. En aimara, se formulan como palabras compuestas, como si se unieran realmente dos lexemas, que en sí son la interpretación de dos voces que dan cuenta de lo que el hablante interpreta como onomatopeya, como se ilustra en los siguientes ejemplos (21). 


\section{(21) COMPUESTOS ONOMATOPÉYICOS}

a. liqi liqi onomatopeya onomatopeya 'ave centinela'

b. puku puku onomatopeya onomatopeya 'gallito de los Andes'

c. q'ixu q'ixu onomatopeya onomatopeya 'trueno, tempestad'

Los hablantes pueden escuchar liqiliqiliqi o q'ixuq'ixuq'ixu, pero su interpretación se da en forma de compuesto reduplicado, es decir, de la unión de dos voces. Las siguientes oraciones ilustran el uso de las onomatopeyas liqiliqi, puku puku y q'ixu q'ixu como unidades composicionales. Estos compuestos se han creado por la reproducción de un sonido y se comportan como una unidad léxico-semántica, como se muestra en (22).

\section{(22) LOS COMPUESTOS ONOMATOPÉYICOS EN UNA ORACIÓN}

a. Janiwa liqiliqiruxa qalampi jaquñati, jallumpiwa irpxatasiri siwa. (refrán)

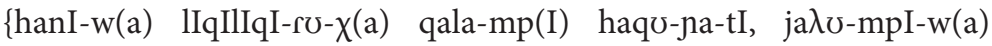
$\operatorname{Irp}(a)-\chi$ ata-sI-rI s(a)-I-wa\} no-FOC liqiliqi-DAT-TOP piedra-INSTR arrojar-NOM-NEG lluviaINSTR-FOC guía-SOB-CONT-AG decir-3S-FOC

'Dicen que no se debe arrojar piedras a los liqi liqi porque suelen traer la lluvia'.

b. Pukupuku-xa chalana arch'ukisi.

\{pokupoku- $\chi($ a) čala-n(a) ar(v)-čr $v k I-s(I)-I\}$

pukupuku-TOP cerro-LOC cantar-FREC-CONT-3S

'El pucu pucu canta en el cerro'.

\footnotetext{
${ }^{4}$ Cotari, Mejía y Carrasco (1978, p. 135) consignan este compuesto de la siguiente forma: “q’ejjo q’ejjo. Rayo, relámpago, trueno / Mä awatirir q’ejjo q’ejjow jiwayatayana. Dice que un rayo ha matado a un pastor / Q'ejjo q'ejj ist'tati? Jalluniwa. ¿Has escuchado el trueno? Va a llover".
} 
c. Q'ixuq'ixu utjki ukjaxa, janiwa muru p'iqi sarnaqañati, jiwañaruwa pursna sapxiwa. (refrán)

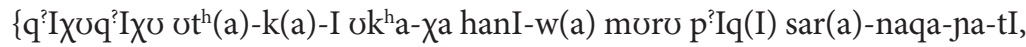
hIwa-na-ro-w(a) por(I)-s(a)-na sa-p $\chi(a)-I-w a\}$

trueno haber-DUR-3S ahí-TOP no-FOC descubierto cabeza caminar-

OSC-NOM-NEG

morir-NOM-IL-FOC caminar-POT-4S decir-PL-3S-FOC

'Dicen que cuando hay truenos, no hay que caminar con la cabeza descubierta porque podemos llegar a morir'.

\subsubsection{Compuestos reduplicados de calidad}

Los compuestos reduplicados de calidad se producen para señalar algunas propiedades específicas de acciones, estado o características de una persona u objeto. Los siguientes casos ilustran este tipo de conceptualización.

\section{(23) Compuestos Reduplicados de CAlidad}

a. thunku thunku

'juego de niños (en saltos, de un solo pie)'

b. nina nina

fuego fuego

'luciérnaga'

c. q'illu q'illu

amarillo amarillo

'arbusto amarillento'

Estos compuestos expresan de alguna manera las características de la acción o estado que se describe. Por ejemplo, thunkuthunku alude a un tipo de juego donde los niños saltan en un solo pie, como se anota en (23a), el cual reduplica el lexema thunku 'salto'. En el caso de ninanina 'luciérnaga', recoge las características de iluminación de nina 'fuego', como se muestra en (23b). Y, en el tercer caso, se tiene en cuenta el color para dar paso al rasgo de un arbusto, como se ve en (23c).

A continuación, se muestra a nivel oracional los compuestos reduplicados que expresan calidad. 
a. Thunkuthunkutha anatiski.

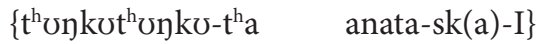

saltos-ABL jugar-PROG-3S

'Está jugando de saltos (de un solo pie)'.

b. Ninaninaxa

arumaki sarnasi.

$\{$ nInanIna- $\chi(\mathrm{a}) \quad$ aroma-kI $\quad \operatorname{sar}(\mathrm{a})-\operatorname{naq}(\mathrm{a})-\mathrm{I}\}$

luciérnaga-TOP noche-LIM andar-OSC-3S

'La luciérnaga solo anda de noche'.

c. Q'illuq'illu Wankani tuqina utji.

$\left\{q^{?} \mathrm{I} \lambda \mho q^{?} \mathrm{I} \lambda \circlearrowright \quad \quad\right.$ wankan(I) $\quad$ toqI-n(a) uth(a)-I\}

planta Huancané UBIC-LOC haber-3S

'El árbol llamado q’illuq'illu existe en Huancané.

\section{CONCLUSIONES}

El aimara es una lengua que presenta compuestos reduplicados, los cuales se crean por el proceso de formación léxica conocido comúnmente como composición; en este caso, se unen dos lexemas con forma y significado similares. En el aimara, la copia reduplicada es siempre una copia completa. Asimismo, esta lengua emplea compuestos reduplicados para formar palabras compuestas básicamente de orden nominal. No hay casos de reduplicaciones para formar verbos u otras categorías léxicas como palabras compuestas.

En el trabajo, hemos manejado la hipótesis de que las reduplicaciones son palabras compuestas en el aimara. Esta parece ser también aceptada por Cerrón-Palomino en 1995:

En lo referente al aimara, la reduplicación completa se da en la formación de palabras compuestas (...). Este fenómeno, según Cerrón-Palomino, "se trata más bien de algunos procesos de composición con identidad léxica nombre+nombre (incluyendo algunas de sus subcategorías). (p. 151, como se citó en Gonzalo, 2011, p. 52)

En este sentido, y por lo que podemos interpretar de sus afirmaciones, ambos autores se inclinan por reconocer también que las reduplicaciones 
en esta lengua se comportan como palabras compuestas. Para los autores, las reduplicaciones completas, es decir donde se juntan dos lexemas iguales, constituyen casos de palabras compuestas. Se trata de composiciones nominales, donde un nombre con forma idéntica se une con otro también de forma idéntica.

Específicamente, las reduplicaciones $\mathrm{N}_{1}+\mathrm{N}_{1}$ son casos de composición morfológica, en los que un mismo elemento se reitera y se adjunta al otro con el fin de crear un nuevo significado. Entonces, en el aimara, los compuestos reduplicados son casos de composición o de una clase de composición.

Hemos visto que los compuestos reduplicados se comportan como palabras compuestas, pues al igual que estas presentan un solo acento, tienen un significado propio, y son unidades con cohesión sintáctica. Por último, los compuestos reduplicados de la lengua refieren a conceptos de intensidad, cantidad, calidad y forma.

Finalmente, se puede argüir que lo que se demuestra es que las palabras reduplicadas son palabras, pero no necesariamente originadas por composición, pues podrían también ser originadas por derivación, si se considera que la reduplicación es un proceso derivativo. Sin embargo, para que se trate de una derivación, la palabra que se reduplica tendría que funcionar como un afijo derivativo, que incluso pueda adjuntarse a casos no reduplicados, como ocurre en los casos comunes de las derivaciones morfológicas, y no comportarse como un lexema independiente, como sí ocurre en la composición de palabras. Como señalamos arriba, Fabb (2001) indica que la reduplicación de palabras completas es descrita como un proceso de composición, puesto que cada parte de la palabra resultante corresponde a una palabra que aparece y se usa de forma independiente en la formación de palabras.

Al respecto, este trabajo contribuye al debate sobre la reduplicación como un caso de composición a partir de la adjunción de segmentos completos. Los estudios posteriores pueden contradecir los presentes postulados y estudiar si las reduplicaciones parciales también pueden por alguna razón considerarse casos de compuestos en lenguas que se comporten como tales. Sobre el aimara, las investigaciones deben continuar retomándose más aun cuando es una de las lenguas andinas no siempre estudiada, al igual que el jaqaru. 


\section{REFERENCIAS}

Adelaar, W. \& Muysken, P. (2004). The languages of the Andes. Cambridge: Cambridge University Press.

Almela, R. (1999). Procedimientos de formación de palabras en español. Barcelona: Ariel.

Alvar, M. (2006). La formación de palabras en español. Madrid: Arco/Libros.

Andrade, L. (2009). Las lenguas indígenas del presente. En L. Andrade y J. Pérez. Las lenguas del Perú (pp. 36-79). Lima: Pontificia Universidad Católica del Perú.

Aronoff, M. (1976). Word formation in generative grammar. Cambridge/MA: MIT Press.

Ayala, J. (1988). Diccionario español. Aymara español. Lima: Juan Mejía Baca.

Bauer, L. (2009). Typology of compounds. En R. Lieber \& P. Štekauer (eds.). The Oxford Handbook of Compounding (pp. 343-356). Oxford: Oxford University Press.

Bertonio, L. ([1612] 1984). Vocabulario de la lengua aymara. Edición facsimilar. Cochabamba: CERES, Instituto Francés de Estudios Andinos (IFEA), Museo Nacional de Etnografía y Folklore (MUSEF).

Booij, G. (2007). The Grammar of Words. An Introduction to Morphology, $2^{\mathrm{a}} \mathrm{ed}$. Oxford: Oxford University Press.

Briggs, L. (1993). El idioma aymara: variantes regionales y sociales. La Paz: ILCA.

Büttner, Th. y Condori, D. (1984). Diccionario aymara-castellano. Arunakan liwru: Aymara-kastillanu. Puno: CORPUNO y Dirección Departamental de Educación/Puno.

Callo, S. (2009). Kamisaraki: diccionario aymara-castellano, castellano-aymara [Kamisaraki: piwra arunaka aymarata kastillanuru qillqata]. $2^{\mathrm{a}}$ ed. Tacna: Caja Municipal Tacna.

Carreño, P. (2006). El quechua y la modernidad: instrumentos para crear un vocabulario actual. RunasimiNet. Lima: RIDEI-Pontificia Universidad Católica del Perú (PUCP).

Carvajal, J., Hernández, A. y Ramos, N. (2001). Diccionario ilustrado aymara, español, inglés. Santiago: Maitén Editores.

Cerrón-Palomino, R. (2000). Lingüística aimara. Cusco: Centro de Estudios Regionales Andinos Bartolomé de las Casas (CBC).

Cerrón-Palomino, R. ([1994] 2008). Quechumara. Estructuras paralelas del quechua y del aimara. La Paz: Universidad Mayor de San Simón (UMSS), PROEIB Andes, Plural Editores.

Cerrón-Palomino, R. y Carvajal, J. (2013). Aimara. En M. Crevels y P. Muysken (eds.). Lenguas de Bolivia (pp. 169-213). La Paz: Centro de Estudios (CLS) de la Universidad de Radboud de Nimega, la organización neerlandesa de investigaciones científicas (NWO), la Real Academia Neerlandesa de Ciencias (KNAW) y la Embajada del reino de los Países Bajos en la Paz. 
Coler, M. (2014). A grammar of Muylaq' Aymara: Aymara as spoken in Southern Peru. Leiden: Brill.

Cotari, D., Mejía, J. y Carrasco, V. (1978). Diccionario aymara-castellano, castellano-aymara. Cochabamba: Instituto de Idiomas Padres de Maryknoll.

De Lucca, M. (1987). Diccionario práctico aymara-castellano/castellano-aymara. La Paz: Los Amigos del Libro.

De Pedro Ricoy, R., Howard, R., Reynoso, R. y Andrade, L. (2020). 'Nosotras le llamamos acompañamiento': las dirigentas quechuas y aimaras del sur peruano y la interpretación ad hoc. Latin American and Caribbean Ethnic Studies 16, 63-84. https://doi.org/10.1080/17442222.2020.1770986

Deza, J. (1989). Jaya mara aru. Nuevo diccionario. Aymara-castellano. Castellano-aymara. Lima: CONCYTEC.

Deza, J. (1992). Gramática de la lengua aymara. Lima: Artex.

Dressler, W. (2007). Compound types. En G. Libben y G. Jarema (eds.). The representation and processing of compound words (pp. 23-44). Oxford: Oxford University Press.

Escandell, V. (1991). Sobre las reduplicaciones léxicas. Separata de 'Lingüística española actual, 13, 71-86.

Fabb, N. (2001). Compounding. En A. Spencer \& A. Zwicky (eds.), The Handbook of Morphology (pp. 66-83). Oxford: Blackwell Publishers.

Gonzalo, R. (2011). La derivación verbal en el aimara de Pomata (Tesis de Maestría en Lingüística con mención en Estudios Andinos). Pontificia Universidad Católica del Perú (PUCP), Lima.

Gonzalo, R. (2018). Derivación de verbos de cambio en el aimara: -pta y -ra. Lengua y Sociedad 17(2), 63-89. Recuperado de http://revista.letras.unmsm.edu.pe/index.php/ls/article/view/1094

Hardman, M., Vásquez, J. y de Dios Yapita, J. (1988). Aymara. Compendio de estructura fonológica y gramatical. La Paz: Gramma.

Haspelmath, M. (2002). Understanding Morphology. Londres: Arnold.

Huayhua, F. (2001). Gramática descriptiva de la lengua aimara (Aymara Aru Yatiwi). Lima: Instituto de Reafirmación de los Pueblos Aimaras Quechuas y Amazonenses.

Huayhua, F. (2009). Diccionario bilingüe polilectal aimara-castellano / castellano-aimara. Lima: Universidad Nacional Mayor de San Marcos (UNMSM).

Inkelas, Sh. (2014). Non-concatenative derivation: Reduplication. En R. Lieber \& P. Štekauer (eds.). The Oxford Handbook of Derivational Morphology (pp. 169-189). Oxford: Oxford University Press.

Inkelas, Sh. y Zoll, Ch. (2005). Reduplication. Doubling in Morphology. Cambridge: Cambridge University Press.

Lang, M. (1992). Formación de palabras en español. Morfología derivativa productiva en el léxico moderno. Madrid: Cátedra.

Lieber, R. \& Štekauer, P. (eds.). (2009). The Oxford Handbook of Compounding. Oxford: Oxford University Press.

Lovón, M. (2009). Hacia una teoría de la complejidad: estudio etnolingüístico 
y cognitivo de la correlación entre los platos típicos tupinos y su construcción lexical en la lengua jaqaru. (Tesis de Licenciatura para obtener el grado de Licenciado en Lingüística). Universidad Nacional Mayor de San Marcos (UNMSM), Lima, Perú.

Lovón, M. (2016). Enseñanza de la lengua vernácula como segunda lengua: Lenguas indígenas peruanas en estado de L2. Escritura y Pensamiento 19(38), 185-210.

Lovón, M. (2018). Análisis crítico del discurso lexicográfico (ACDL): un examen a la historia de las lenguas andinas en los diccionarios de la RAE. Boletín de la Academia Peruana de la Lengua 64(64), 141-172. https://doi. org/10.46744/bapl.201802.008

Lovón, M. (2019). Las palabras compuestas en el aimara de Conima. Lexis 43(1), 55-85. https://doi.org/10.18800/lexis.201901.002

Lovón, M. (2020). “La literacidad para legislar”: Una creencia hegemónica de la literacidad reproducida por el diario el Correo. Literatura y Lingüística, 41, 413-454. https://doi.org/10.29344/0717621X.41.2271

Lovón, M. y Quispe, A. (2020). ¿Quién tiene el derecho a opinar sobre política lingüística en el Perú?: Un análisis crítico del discurso. Íkala. Revista de Lenguaje y Cultura 25(3), 733-751. https://doi.org/10.17533/udea.ikala. $\mathrm{v} 25 \mathrm{n} 03 \mathrm{a} 12$

Plag, I. (2003). Word Formation in English. Cambridge: Cambridge University.

Plag, I. \& Winther, L. (2016). Derivational morphology: An integrative perspective on some fundamental questions. En V. Pirelli, I. Plag, \& W. Dressler (eds.), Word knowledge and word usage: A crossdisciplinary guide to the mental lexicon (pp. 295-335). Berlin y New York: De Gruyter.

Roca, F. y Suñer, A. (1998). Reduplicación y tipos de cuantificación en español. Estudi General, 17, 37-66. Recuperado de https://www.raco.cat/index.php/ EstudiGral/article/view/43747/56177

Sánchez, J. (2009). La formación de palabras por composición. Revista de Filología Española 59(1), 103-128.

Spencer, A. \& Zwicky, A. (2001). The Handbook of Morphology. Oxford: Blackwell.

Štekauer, P., Valera, S., \& Körtvélyessy, L. (2012). Word-Formation in the World's Language. Cambridge: Cambridge University Press.

Varela, S. (2005). Morfología léxica: La formación de palabras. Madrid: Gredos.

Weber, D. ([1989] 1996). Una gramática del quechua del Huallaga. Traducción de Marlene Ballena Dávila. Lima: Ministerio de Educación e Instituto Lingüístico de Verano. 


\section{ANEXO: LISTA DE SÍMBOLOS Y ABREVIATURAS}

1S primera persona del singular

2P segunda persona del singular

3S tercera persona del singular

$4 \mathrm{~S} \quad$ cuarta persona del singular

$\mathrm{N}$ nombre

ADJ adjetivo

PL plural

AG agentivo

AC acusativo

ASC ascensor

ATR atributivo

ABL ablativo

BEN benefactivo

CL cislocativo

CONT continuativo

DAT dativo

DUR durativo

FREC frecuentativo

IL ilativo

INCD inceptivo

INSTR instrumental

LOC locativo

NEG negación

NOM nominativo

PROG progresivo

OSC oscilativo

POT pontencial

SOB sobrecolocador

UBIC ubicativo

FOC focalizador

TOP topicalizador

C consonante

$\mathrm{V}$ vocal

, acento

$\rightarrow \quad$ da forma a

[ ] representación fonética

\{\} representación morfológica

// representación fonológica

$<$ grafía

', glosas

- $\quad$ separación morfológica

$+\quad$ adición 
- $\quad$ separación silábica

* forma agramatical

( ) elisión

$/ \mathrm{I} / \quad<\mathrm{i}>\quad$ vocal alta anterior

$/ \mathrm{v}<\mathrm{u}>$ vocal alta posterior

$/ a /<a>$ vocal baja central

$/ \mathrm{p} / \quad<\mathrm{p}>\quad$ consonante oclusiva bilabial simple

$/ \mathrm{p}^{\text {? }}\left\langle\mathrm{p}^{\prime}\right\rangle$ consonante oclusiva bilabial glotalizada

$/ \mathrm{p}^{\mathrm{h}} /<\mathrm{ph}>$ consonante oclusiva bilabial aspirada

/t/ $\quad<\mathrm{t}\rangle \quad$ consonante oclusiva alveolar simple

$/ \mathrm{t}^{\mathrm{2}} / \quad\left\langle\mathrm{t}^{\prime}\right\rangle \quad$ consonante oclusiva alveolar glotalizada

$/ \mathrm{t}^{\mathrm{h}} /<\mathrm{th}>$ consonante oclusiva alveolar aspirada

$/ \mathrm{k} / \quad<\mathrm{k}>\quad$ consonante oclusiva velar simple

$\left./ \mathrm{k}^{?} / \quad<\mathrm{k}\right\rangle$ consonante oclusiva velar glotalizada

$/ \mathrm{k}^{\mathrm{h}} / \quad<\mathrm{kh}>$ consonante oclusiva velar aspirada

/q/ $<\mathrm{q}>$ consonante oclusiva postvelar simple

$/ q^{?} / \quad<q^{\prime}>$ consonante oclusiva postvelar glotalizada

$/ \mathrm{q}^{\mathrm{h}} /<\mathrm{qh}>$ consonante oclusiva postvelar aspirada

/čl <ch $>$ consonante africada palatal simple

|č? $/ \quad<$ ch'> consonante africada palatal glotalizada

$/ \check{c}^{h} /<\mathrm{chh}>$ consonante africada palatal aspirada

/s/ $\quad<s>$ consonante fricativa alveolar

$|\chi|<x>\quad$ consonante fricativa postvelar

$/ \mathrm{h} / \quad<\mathrm{j}>\quad$ consonante fricativa glotal

$/ \mathrm{m} /<\mathrm{m}>$ consonante nasal bilabial

$/ \mathrm{n} / \quad<\mathrm{n}>\quad$ consonante nasal alveolar

$/ \mathrm{n} / \quad<\tilde{\mathrm{n}}>\quad$ consonante nasal palatal

/l/ $\quad<\mathrm{l}>\quad$ consonante lateral alveolar

$/ \lambda /<$ ll $>$ consonante lateral palatal

$\mid \mathrm{r} / \quad<\mathrm{r}>\quad$ consonante vibrante simple

$/ \mathrm{w} / \quad<\mathrm{w}\rangle$ semiconsonante bilabial

$/ y /<y>\quad$ semiconsonante palatal 